\title{
Formal and Empirical Methods in Philosophy of Science
}

\author{
Vincenzo Crupi ${ }^{\dagger}$ and Stephan Hartmann ${ }^{\dagger}$
}

\begin{abstract}
This essay addresses the methodology of philosophy of science and illustrates how formal and empirical methods can be fruitfully combined. Special emphasis is given to the application of experimental methods to confirmation theory and to recent work on the conjunction fallacy, a key topic in the rationality debate arising from research in cognitive psychology. Several other issue can be studied in this way. In the concluding section, a brief outline is provided of three further examples.
\end{abstract}

\section{Introduction}

Philosophers of science use a plurality of apparently divergent methods. This claim can easily be substantiated by looking into one of the relevant journals: one realizes that some authors use the traditional method of conceptual analysis, other engage in formal modelling, conduct case studies and - more recently - experiments, or consult the history of science in considerable detail. But how do these methods relate to each other? Is one of them the right one?

Pluralistic cautions would suggest that multiple methodological approaches are legitimate. In fact, we would like to stress that a combination of two or more methods may be particularly fruitful in some cases. Carnap, for example, combined formal methods (i.e., logic and probability theory) with conceptual analysis to arrive at an explication of the notion of confirmation. And authors in the tradition of Kuhn and Feyerabend use case studies from the history of science to challenge philosophical models of scientific reasoning such as Popper's falsificationism. In this essay we would like to explore how formal methods and experiments can be combined.

Experiments are all the rage in contemporary philosophy (Knobe and Nichols 2008, Stotz 2009). In epistemology, people's intuitions about Gettier cases have famously been tested. In ethics, aspects of the freedom of will debate are studied experimentally. Philosophers of language also test our intuitions about the reference of proper names. This list could easily be continued. Interestingly, the results of these studies are often surprising when compared with the corresponding intuitions of professional philosophers.

\footnotetext{
$\dagger$ Contact information: Vincenzo Crupi, Department of Philosophy, University of Turin, via Sant'Ottavio 20, 10124 Turin (Italy), email: vincenzo.crupi@unito.it.

$\$$ Contact information: Stephan Hartmann, Tilburg Center for Logic and Philosophy of Science, Tilburg University, 5000 LE Tilburg, email: S.Hartmann@uvt.nl.
} 
While many of these experiments are used to test philosophers' intuitions (or hypotheses), it is worth noting that experiments have other functions besides testing, as Hacking (1983) reminded us. Experiments may, for example, inspire new hypotheses, and this holds for experimental research in traditional domains as well as in philosophy. Usually, these hypotheses are not put forward in a theoretical vacuum: they may relate to an existing theoretical framework, and so some tinkering may have to be done to fit the new hypothesis (or a modified version of it) into the theoretical framework (or a modified version of it). In short, experimental data may provide guidance and insight in theory-construction in a number of ways.

This essay is meant to illustrate the claims above. It focuses on experiments and experimental phenomena which are directly related to work done by formal epistemologists. More specifically, we will look at two case studies. Section 2 focuses on confirmation theory and the recent empirical work in this field. Section 3 discusses the conjunction fallacy, which is of considerable importance as the rationality debate lurks in the background. Finally, we will outline a list of open problems suggesting promising lines of research to be pursued further in the future.

\section{Case-study I: Confirmation}

Hypothesis testing and confirmation have been central issues in the philosophy of science for decades. Early accounts based on logic and essentially qualitative notions have struggled to deal with a number of puzzles, including the "tacking" problem, Hempel's paradoxes, Goodman's new riddle, the variety of evidence, and the DuhemQuine thesis. Importantly, such issues have been shown to receive a more effective treatment in quantitative terms within a Bayesian approach to confirmation and scientific reasoning (see Earman 1992, pp. 63-86, for a now classical discussion in this vein). A quantitative approach also seems to be up to a general real-world challenge: judgments concerning the amount (or degree) of support that a piece of information brings to a hypothesis are commonly required in scientific research as well as in other domains (medicine, law). Thus, a central aim of philosophy of science and epistemology is to provide a proper foundation to such judgments.

Bayesianism arguably is a major theoretical perspective in contemporary discussions of reasoning in science as well as in other domains (e.g., Bovens and Hartmann 2003, Howson and Urbach 2006, Oaksford and Chater 2007). Bayesian theorists postulate a probabilistic analysis of many sorts of ordinary and scientific reasoning by endorsing a subjective reading of probability, i.e., by using probabilities to model degrees of subjective belief. Within this framework, contemporary Bayesians commonly identify confirmation with an increase in the probability of a hypothesis $h$ provided by a piece of evidence $e$ as compared to the initial probability of $h$ (i.e., with evidence $e$ not being given). A natural way to measure confirmational strength then amounts to a function mapping relevant probability values of $h$ and $e$ onto a number which is either positive, null or negative depending on $p(h \mid e)$ being higher, equal or lower as compared to $p(h)$. Among traditional proposals meeting this basic constraint are the following: ${ }^{1}$

\footnotetext{
${ }^{1}$ These classical measures trace back to Carnap (1950/1962, p. 361), Keynes (1921, pp. 150-155) and Alan Turing (as reported by Good, 1950, pp. 62-63), respectively.
} 
- the difference measure: $D(h, e)=p(h \mid e)-p(h)$

- the (log) ratio measure: $R(h, e)=\log [p(h \mid e) / p(h)]$

- the $(\log )$ likelihood ratio measure: $L(h, e)=\log [p(e \mid h) / p(e \mid \neg h)]$

More recent variants include the following: ${ }^{2}$

$$
\begin{aligned}
& S(h, e)=p(h \mid e)-p(h \mid \neg e) \\
& Z(h, e)= \begin{cases}\frac{p(h \mid e)-p(h)}{1-p(h)} & \text { if } p(h \mid e) \geq p(h) \\
\frac{p(h \mid e)-p(h)}{p(h)} & \text { if } p(h \mid e)<p(h)\end{cases}
\end{aligned}
$$

Quantitative Bayesian accounts of confirmation can usefully merge with (and profit from) various technical and theoretical refinements and extensions of the Bayesian framework, such as the use of Bayesian networks and probability updating upon uncertain evidence (e.g., Crupi, Festa and Mastropasqua, 2008). Importantly, quantitative measures such as those listed above allow ordinal judgments concerning confirmational strength, such as: "hypothesis $h$ receives more empirical support by $e_{1}$ than by $e_{2}$ " or " $e$ confirms $h_{1}$ to a greater extent than $h_{2}$ ". One open problem here is that - as both Fitelson (1999) and Festa (1999) emphasized - alternative confirmation measures are not generally ordinally equivalent (for a proof concerning a whole set of measures including the five above, see Crupi, Tentori and Gonzalez 2007, p. 231). Indeed, their implied rankings crucially diverge in various interesting classes of cases. This is known as the "problem of measure sensitivity" (Fitelson 1999).

In philosophical quarters, two opposite kinds of reactions can be identified concerning the plurality of confirmation measures (Steel 2007). On the one hand, one can bite the bullet and take a largely pluralistic stance: different measures reflect different aspects of the confirmation relation (e.g., Joyce 2004; Huber 2008; also see Crupi, Festa and Buttasi forthcoming). So far, however, it is not quite clear how this form of pluralism relates to actual scientific practice and real-world judgments of confirmational strength. On the other hand, one may want to argue in favour of the specific properties of one particular measure on independent, often intuitive, grounds. As a matter of fact, though, intuitions diverge among scholars. So much so that various conflicting measures have been defended in this way (see, for instance, Milne 1996, and Fitelson 2001).

Interestingly, the plurality of confirmation measures has been addressed empirically in recent times, fostering a novel line of experimental investigation in the psychology of reasoning. The basic idea has been to see whether and how empirical data of naïve reasoners' judgments sort out alternative proposals from the literature in philosophy of science and formal epistemology. Two recent papers (Tentori et al. 2007; Crupi,

\footnotetext{
${ }^{2}$ Measure $S(h, e)$ has been independently introduced by Christensen (1999) and Joyce (1999). As pointed out in Crupi, Festa and Buttasi (forthcoming), $Z(h, e)$ can be seen as a measure of the relative reduction of uncertainty. It has been explicitly advocated by Crupi, Tentori and Gonzalez (2007). Other occurrences include Rescher (1958, p. 87), Shortliffe and Buchanan (1984, pp. 248 ff.), Cooke (1991, p. 57) and Mura $(2006,2008)$.
} 
Tentori and Gonzalez 2007) report results from the first attempts to test the descriptive adequacy of alternative Bayesian measures of confirmation with an urn setting experiment and naïve participants (university students). The results seem highly interesting. To begin with, this study provides the first neat demonstration that probabilistic confirmation (as contrasted to probability tout court) does belong to the repertoire of the human mind. Second, it shows that the theoretical divergence among confirmation measures is of psychological significance, as competing accounts do yield different degrees of predictive accuracy. In particular, measure $Z$ scored as the most accurate predictor of elicited confirmation judgments, with a slight advantage over the theoretically appealing competitors of likelihood ratio based measures. Crupi, Tentori and Gonzalez (2007) also presented normative reasons in favour of $Z$, suggesting that it might eventually be singled out on descriptive and normative grounds alike. (It should be noticed that a rather similar scenario is emerging with regards to another sophisticated concept from contemporary Bayesian epistemology, which is also strictly connected to confirmation, i.e., coherence. We'll come back to the latter issue in Section 4. below)

The interaction with formal philosophy of science also extends to more traditional branches of experimental research in cognitive psychology. A case in point is represented by the "selection task" (Wason 1966, 1968). Since the very beginning, this widely known experimental paradigm was directly inspired by earlier accounts of hypothesis testing in the philosophy of science. And indeed, cognitive psychologists have recently stressed and explored tight connections with Hempel's celebrated raven paradox (McKenzie and Mikkelsen 2000). For long thought to elicit a basic form of "confirmation bias" and irrational behaviour (see, e.g., Manktelow and Over 1993, Stich 1990, Stein 1996), the selection task has then been reanalyzed through a sophisticated Bayesian account of information search, by which participants' responses have been said to be not only vindicated, but also actually explained as arising from cognitive processes reflecting rational data selection (Oaksford and Chater 1994; see Oaksford and Chater 2003, and the references therein for major contributions to the lively debate on this issue). Indeed, models of the value of information (see Nelson 2005) yield important theoretical connections with probabilistic confirmation. So much so that other similar accounts of the same experimental phenomenon explicitly resort to standard Bayesian measures of confirmation (Nickerson 1996, and Fitelson forthcoming). Finally, Bayesian confirmation measures have also occurred in debates on normative and behavioural aspects of "probative value" in legal contexts (see Davis and Follette 2002, 2003, and Kaye and Koehler 2003) as well as in the psychological literature on causal induction (see Perales and Shanks 2003).

\section{Case-study II: The Conjunction Fallacy}

The final remarks in the previous section document a growing trend to rely on tools from formal epistemology and philosophy of science for a better insight into long standing phenomena and puzzles in the empirical study of human cognition. A further rich source of relevant considerations arises from the recent literature on yet another largely known phenomenon: the conjunction fallacy. In an often quoted illustration, Tversky and Kahneman (1983) had participants faced with the description of a character, Linda (31 years old, single, outspoken and very bright, with a major in 
philosophy and concerns about discrimination, social justice and pacifism), ranking the conjunction "Linda is a bank teller and is active in the feminist movement" as more probable than "Linda is a bank teller". From then on, a number of studies have reported that, under certain conditions, people may judge a conjunction of hypotheses as more probable than one of its conjuncts, contrary to elementary principles of probability theory.

The conjunction fallacy has become a key topic in debates on the rationality of human reasoning and its limitations. The phenomenon prompted an enormous amount of research work in psychology and beyond, like many others achievements from Tversky and Kahneman's research programme, which readily and steadily attracted interest from philosophers (Levi 1985; Stich 1990; Samuels, Stich and Bishop 2002) and also established the new interdisciplinary field of behavioural economics (Camerer, Loewenstein and Rabin 2003).

For more than two decades, psychologists have discussed and empirically explored the subtleties of the conjunction fallacy effect, ultimately showing that its robustness and recurrence deserve explanation in a satisfactory account of human reasoning and judgment (see Sides et al. 2002; Tentori, Bonini and Osherson 2004; Wedell and Moro 2008).

A formal analysis accounting for results obtained in the Linda problem has been presented by Bovens and Hartmann (2003, pp. 85-88). Briefly put, the proposal is the following. Suppose "Linda is a bank teller" and "Linda is a feminist bank teller" are reports of two distinct sources of information $s_{1}$ and $s_{2}$ which are not perfectly reliable. Linda's description may well suggest that source $s_{1}$ is less reliable than $s_{2}$. But then, probability theory is consistent with the statement that the probability of "bank teller" conditional on the relatively low reliability of $s_{1}$ is lower than the probability of "feminist bank teller" conditional on the relatively high reliability of $s_{2}$. Bovens and Hartmann (2003) submit that this is what participants' responses express. (See Hintikka 2004, for an independent argument along similar lines). More recently, Hartmann and Meijs (forthcoming) provided a more sophisticated variant of this account, and plan to put it to empirical test.

A different approach has been taken by Crupi, Fitelson and Tentori (2008), following and extending some earlier suggestions from both the psychological and philosophical literature (e.g., Tenenbaum and Griffiths 2001; Levi 2004). While recognizing that conjunction fallacy results document a genuine error in probabilistic judgment, these authors have outlined an explanatory framework based on the notion of confirmation, meant in terms of Bayesian confirmation theory (see Section 2. above). By a close analysis of previous empirical results (Osherson et al. 1990, and Lagnado and Shanks 2002), they argued that the participants' fallacious probability judgments might reflect the assessment of confirmation relations among the evidence provided and the hypotheses at issue in the experimental scenarios. Moreover, extending an earlier result by Sides et al. (2002), they showed that, in a whole class of cases including the Linda example along with others, Bayesian quantitative models of inductive confirmation imply that the evidence provided does support the conjunctive statement more than the single conjunct. Roughly, this class of cases is identified by the evidence provided (e.g., Linda's description) confirming the added conjunct ("feminist") but not the isolated one ("bank teller") (see Tentori and Crupi 2009, for 
original data in support of this account; Tentori and Crupi forthcoming, and Schupbach forthcoming for a debate; and Atkinson, Peijnenburg, and Kuipers 2009 for some further relevant results).

The latter confirmation-theoretic reading of the Linda problem is one way to flesh out the otherwise esoteric statement by Tversky and Kahneman themselves that "feminist bank teller is a better hypothesis about Linda than bank teller" (1983, p. 311). A different strategy to fill in the blanks of this noteworthy remark has been provided Cevolani, Crupi and Festa (forthcoming), suggesting that assessments of expected verisimilitude may also crucially contribute to conjunction fallacy results. Indeed, these authors proved that, under very weak and plausible assumptions, "feminist bank teller", while less likely to be true than "bank teller", may well be more likely to be close to the whole truth about Linda in a well-defined formal sense. As it can be seen, proposed explanations of the conjunction fallacy based on core notions from the philosophy of science have literally flourished in recent times. For a further example, Shogenji (forthcoming) should be mentioned, who employs a probabilistic and quantitative theory of epistemic justification to account for the phenomenon.

\section{Open Problems and concluding remarks}

In this section, we sketch three open problems from the philosophy of science that might well gain from a combination of formal and empirical methods.

a. Justification and Coherence. According to the coherence theory of justification, a set of propositions (e.g., a scientific theory) is justified if the respective propositions cohere with each other. But what does it mean that propositions cohere with each other? And how can one measure how much they cohere? To address these questions, various measures of coherence have been proposed (Bovens and Hartmann 2003, Douven and Meijs 2007). However, there is no consensus in the literature as to what the right measure is: different authors appeal to different intuitions or stress different formal requirements, and none of the measures on the market satisfies all of them. This, in turn, lead to a deadlock of the debate, which needs to be resolved. Inspired by the successful work on confirmation measures (see Section 2), empirical investigation seems to provide a promising perspective. It will help us to understand better which role coherence plays in people's actual judgments, and which (if any) of the proposed measures is psychologically realistic. Empirical studies may also foster the construction of alternative measures, or the refinement of existing ones. Notably, original experimental procedures to compare different quantitative accounts of coherence have been recently devised by Harris and Hahn (forthcoming).

b. Scientific explanation. The debate about scientific explanation is in a similar situation. Here we find a spectrum of different theories, supported by altogether different philosophical background beliefs. In this situation, empirical studies may stir the debate in a new direction. More specifically, the following philosophically relevant questions seem to be worth addressing experimentally: Which role do simplicity, probability and coherence play in explanations? And how do people assess the strength of an explanation? The resulting findings will help evaluating existing theories of explanation and may inspire new ones. For some preliminary work in this direction, see Lombrozo (2006) and Schupbach and Sprenger (2009). 
c. Social epistemology and philosophy of science. Social epistemology studies the social aspects of science from an epistemological point of view. To do so, case studies have been conducted and formal models have been constructed (see, e.g., Lehrer and Wagner 1981, and Hartmann, Martini and Sprenger 2009). These studies should be accompanied by empirical investigations, as some "empirical input" is needed to answer questions such as the following: What is the best way to proceed when different scientists disagree? Philosophers and decision theorists developed a host of models that reflect certain ideals of rationality. These models are typically a priori, i.e., they do not include any empirical information about the deliberation process. Conducting experiments will help us to better understand how deliberation works and, eventually, how deliberation should work if the goal of the committee in question is to make the right decision.

Most of the examples above illustrate ongoing trends of research, fostering (and requiring) much further work to provide fully established results. For our present purposes, however, two connected remarks can be firmly put forward. First, the theoretical toolbox of researchers empirically investigating human cognition and behaviour is being expanded from basic probability theory to more advanced formal notions with distinct philosophical origins. Second, a number of recent and current empirical investigations have a potential to provide a fresh look on traditional concerns addressed by formal epistemology and philosophy of science.

\section{Acknowledgements}

Work supported by a grant from the Spanish Department of Science and Innovation (FFI2008-01169/FISO). 


\section{References}

Atkinson, D., Peijnenburg, J., and Kuipers, T. (2009), "How to confirm the conjunction of two disconfirmed hypotheses", Philosophy of Science, 76, pp. 1-21.

Bovens, L. and Hartmann, S. (2003), Bayesian epistemology, Oxford University Press, Oxford (UK).

Camerer, C., Loewenstein, G. and Rabin, M. (2003), Advances in Behavioral Economics, Princeton University Press, Princeton (NJ).

Carnap, R. (1950/1962), Logical Foundations of Probability, University of Chicago Press, Chicago.

Cevolani, G., Crupi, V., and Festa, R. (forthcoming), "The whole truth about Linda: Probability, verisimilitude, and a paradox of conjunction", in C. Sinigaglia and M. D'Agostino (eds.), Selected papers from the 2007 Conference of the Italian Association for Logic and Philosophy of Science, College Publications, London.

Christensen, D. (1999), "Measuring Confirmation", Journal of Philosophy, 96, pp. 437-461.

Cooke, R.M. (1991), Experts in Uncertainty. Opinion and Subjective Probability in Science, Oxford University Press, Oxford (UK).

Crupi, V., Festa, R. and Buttasi, C., "Towards a grammar of confirmation", in M. Dorato, M. Rèdei and M. Suárez (eds.), Selected papers from the First Conference of the European Association for the Philosophy of Science, Springer, Berlin.

Crupi, V., Festa, R. and Mastropasqua, T., "Bayesian confirmation by uncertain evidence: A reply to Huber (2005)", The British Journal for the Philosophy of Science, 59 (2008), pp. 201-211.

Crupi, V., Fitelson, B. and Tentori, K. (2008), "Probability, confirmation and the conjunction fallacy", Thinking and Reasoning, 14, pp. 182-199.

Crupi, V., Tentori, K. and Gonzalez, M. (2007), "On Bayesian measures of evidential support: Theoretical and empirical issues", Philosophy of Science, 74, pp. 229-252.

Davis, D. and Follette, W.C. (2002), "Rethinking probative value of evidence: Base rates, intuitive profiling and the postdiction of behavior", Law and Human Behavior, 26, pp. $133-158$.

Davis, D. and Follette, W.C. (2003), "Towards an empirical approach to evidentiary ruling", Law and Human Behavior, 27, pp. 661-684.

Douven, I. and Meijs, W. (2007), "Measuring coherence", Synthese, 156, pp. 405-425.

Earman, J. (1992), Bayes or Bust?, Mit Press, Cambridge (MA).Joyce, J. (1999), The Foundations of Causal Decision Theory, Cambridge University Press, Cambridge (UK).

Festa, R. (1999). "Bayesian Confirmation”, in M. Galavotti \& A. Pagnini (eds.), Experience, Reality, and Scientific Explanation, Dordrecht, Kluwer, pp. 55-87.

Fitelson, B. (1999), "The plurality of Bayesian measures of confirmation and the problem of measure sensitivity", Philosophy of Science, 66, pp. S362-S378.

Fitelson, B. (2001), "A Bayesian account of independent evidence with applications", Philosophy of Science, 68, pp. S123-40.

Fitelson, B. (forthcoming), "Bayesian confirmation theory and the Wason selection task", Synthese.

Good, I.J. (1950), Probability and the Weighing of Evidence, Griffin, London.

Hacking, I. (1983), Representing and Intervening, Cambridge University Press, Cambridge (MA).

Harris, A.J.L. and Hahn, U. (forthcoming), "Bayesian rationality in evaluating multiple testimonies: Incorporating the role of coherence", Journal of Experimental Psychology: Learning, Memory, and Cognition.

Hartmann, S. and Meijs, W. (forthcoming) "Walter the banker: The conjunction fallacy reconsidered", Synthese.

Hartmann, S., Martini, C. and Sprenger, J. (2009), "Consensual decision-making among epistemic peers", Episteme, 6, pp. 110-129. 
Hintikka, J. (2004), “A fallacious fallacy?”, Synthese, 140, pp. 25-35.

Howson, C. and Urbach, P. (2006), Scientific Reasoning: The Bayesian Approach, Open Court, La Salle.

Huber, F. (2008), "Milne's argument for the log-ratio measure", Philosophy of Science, 75, pp. 413-420.

Joyce, J. (1999), The Foundations of Causal Decision Theory, Cambridge University Press, Cambridge (UK).

Joyce, J. (2004), "Bayes's theorem", in E. N. Zalta (ed.), The Stanford Encyclopedia of Philosophy (Summer 2004 Edition), URL = http://plato.stanford.edu/archives/sum2004/ entries/bayes-theorem/.

K. Stotz (ed.) (2009), Experimental Philosophy and Philosophy of Science, in Studies in History and Philosophy of Science A, 40 (Special Issue).

Kaye, D.H. and Koehler, J.J. (2003), "The misquantification of probative value", Law and Human Behavior, 27, pp. 645-659.

Keynes, J. (1921), A Treatise on Probability, Macmillan, London.

Knobe, J. and Nichols, S. (2008), Experimental Philosophy, Oxford University Press, New York.

Lagnado, D.A. and Shanks, D.R. (2002), "Probability judgment in hierarchical learning: A conflict between predictiveness and coherence", Cognition, 83, pp. 81-112.

Lehrer, K. and Wagner, C. (1981), Rational consensus in science and society: A philosophical and mathematical study, Reidel, Dordrecht.

Levi, I. (1985), "Illusions about uncertainty", British Journal for the Philosophy of Science, 36 , pp. 331-340.

Levi, I. (2004). Jaakko Hintikka. Synthese, 140, pp. 37-41.

Lombrozo, T. (2006), "The structure and function of explanations", Trends in Cognitive Sciences, 10, pp. 464-470.

Manktelow, K.I., and Over, D.E. (1993), Rationality: Psychological and philosophical perspectives, Routledge, London.

McKenzie, C.R.M. and Mikkelsen, L.A. (2000), "The psychological side of Hempel's paradox of confirmation", Psychonomic Bulletin \& Review, 7, pp. 360-366.

Milne, P. (1996), " $\log [p(h / e b) / p(h / b)]$ is the one true measure of confirmation", Philosophy of Science, 63, pp. 21-6.

Mura, A. (2006), "Deductive probability, physical probability and partial entailment", in M. Alai and G. Tarozzi (eds.), Karl Popper Philosopher of Science, Rubbettino, Soveria Mannelli, pp. 181-202.

Mura, A. (2008), "Can logical probability be viewed as a measure of degrees of partial entailment?", Logic \& Philosophy of Science, 6, pp. 25-33.

Nelson, J.D. (2005), "Finding useful questions: On Bayesian diagnosticity, probability, impact and information gain", Psychological Review, 112, pp. 979-999.

Nickerson, R.S. (1996), "Hempel's paradox and Wason's selection task: Logical and psychological puzzles of confirmation", Thinking and Reasoning, 2, pp. 1-31.

Oaksford, M. and Chater, N. (1994), "A rational analysis of the selection task as optimal data selection", Psychological Review, 101, pp. 608-631.

Oaksford, M. and Chater, N. (2003), "Optimal data selection: Revision, review, and reevaluation", Psychonomic Bulletin \& Review, 10, pp. 289-318.

Oaksford, M. and Chater, N. (2007), Bayesian Rationality: The Probabilistic Approach to Human Reasoning, Oxford University Press, Oxford (UK).

Osherson, D.N., Smith, E.E., Wilkie, O., Lopez, A. and Shafir, E. (1990), "Category-based induction", Psychological Review, 97, pp. 185-200.

Perales, J.C. and Shanks, D.R. (2003), "Normative and descriptive accounts of the influence of power and contingency on causal judgement", Quarterly Journal of Experimental Psychology, 56A, pp. 977-1007.

Rescher, N. (1956), “A theory of evidence”, Philosophy of Science, 25, pp. 83-94. 
Samuels, R., Stich, S. and Bishop, M. (2002), "Ending the rationality wars: How to make disputed about human rationality disappear", in E.Renee (ed.), Common sense, reasoning and rationality, Oxfor University Press, New York, pp. 236-268.

Schupbach J. (forthcoming), "Is the conjunction fallacy tied to probabilistic confirmation?", Synthese.

Schupbach, J. and Sprenger, J. (2009), "The logic of explanatory power", Working Paper, Tilburg Center for Logic and Philosophy of Science.

Shogenji, T. (forthcoming), "The degree of epistemic justification and the conjunction fallacy, Synthese.

Shortliffe, E.H. and Buchanan, B.G. (1984), "A model of inexact reasoning in medicine", in B.G. Buchanan and E.H. Shortliffe (eds.), Rule-Based Expert Systems, Wesley, Reading (MA), pp. 233-262.

Sides, A., Osherson, D., Bonini, N., and Viale, R. (2002), "On the reality of the conjunction fallacy", Memory \& Cognition, 30, pp. 191-198.

Steel, D. (2007), "Bayesian confirmation theory and the likelihood principle", Synthese, 156, pp. $55-77$.

Stein, E. (1996), Without good reason, Oxford University Press, Oxford (UK).

Stich, S. (1990), The fragmentation of reason, MIT Press, Cambridge (MA).

Tenenbaum, J.B. and Griffiths, T.L. (2001), "The rational basis of representativeness", in J.D. Moore and K. Stenning (eds.), Proceedings of 23rd Annual Conference of the Cognitive Science Society, pp. 1036-1041.

Tentori, K. and Crupi, V. (2009), "Explaining the conjunction fallacy: Probability vs. confirmation", Annual Conference of the Cognitive Science Society 2009.

Tentori, K. and Crupi, V. (forthcoming), "How the conjunction fallacy is tied to probabilistic confirmation: Some remarks on Schupbach (2009)", Synthese.

Tentori, K., Bonini, N. and Osherson, D. (2004), "The conjunction fallacy: A misunderstanding about conjunction?", Cognitive Science, 28, 467-477.

Tentori, K., Crupi, V., Bonini, N. and Osherson, D. (2007), "Comparison of confirmation measures", Cognition, 103, pp. 107-119.

Tversky, A. and Kahneman, D. (1983), "Extensional vs. intuitive reasoning: The conjunction fallacy in probability judgment", in T. Gilovich, D. Griffin, and D. Kahneman (eds.), Heuristics and Biases: The Psychology of Intuitive Judgment, Cambridge University Press, New York, pp. 19-48.

Wason, P. (1966), "Reasoning”, in B. Foss (ed.), New Horizons in Psychology, Penguin, Harmonsworth (UK), pp. 135-151.

Wason, P. (1968), "Reasoning about a rule", Quarterly Journal of Experimental Psychology, 20, pp. 273-281.

Wedell, D.H. and Moro, R. (2008), "Testing boundary conditions for the conjunction fallacy: Effects of the response mode, conceptual focus, and problem type", Cognition, 107, pp. 105-136. 